\title{
A Simplified Analysis of the Post-buckling Behavior of a Compressed Reinforcing Bar
}

P. Kabele

Recently, a computational methodology based on a sequential multiscale approach, which facilitates numerical simulation of an $R / C$ building demolition has been developed. In this type of analysis, it is necessary to capture the behavior of compressed reinforcement bars until complete rupture, which occurs due to extensive bending in the post-buckling regime. To this end, a simplified analytical model of the post-buckling behavior of a compressed bar is proposed. The simplification consists namely in considering rigid-plastic material behavior, neglecting axial contraction of the central line, and approximating the shape of the deformed central line in the plastic hinges by a circular arch. Consequently, the axial loading force, bar end displacement, and extreme strain can be expressed in relatively simple closed forms. The results obtained with the proposed model show very close agreement with those obtained by a detailed and realistic finite element analysis, which justifies the use of the simplifying assumptions.

Keywords: reinforcement bar, hardening plasticity, post-buckling behavior.

\section{Introduction}

When a precast reinforced concrete $(\mathrm{R} / \mathrm{C})$ panel building is demolished using controlled explosions, selection of appropriate sizes, placement, and timing of the charges is crucial in order to ensure complete collapse of the structure while not damaging surrounding objects. The design of safe and efficient deconstruction procedures can be facilitated by means of computer simulations. In contrast to the standard structural analysis, the main objective of such a simulation is to predict the mechanical behavior of a structure during the phase when it disintegrates and loses static stability. The mechanical phenomena to be dealt with include dynamic motion (finite displacements and rotations) and interaction of debris on the structural level (macroscale), and fracture and yielding on the material level (mesoscale). Simultaneous treatment of all these mechanisms would be computationally too costly; thus, a computational methodology based on a sequential multiscale approach has recently been proposed [1]. In order to reduce the number of degrees of freedom involved in the dynamic analysis at the macroscale, the entire structure is modeled as an assembly of beam finite elements, which represent structural members (panels) and their joints. The governing relations among bending moment, axial force, curvature, and axial strain of the beam elements are formulated by modeling the overall behavior of $\mathrm{R} / \mathrm{C}$ panel sections or joints that undergo local damage (mesoscale model).

During demolition, structural members and joints may be exposed to loading states that are diametrically different from those for which they were designed. Nevertheless, the load bearing capacity of an $\mathrm{R} / \mathrm{C}$ section exposed to an arbitrary combination of axial force and bending is usually determined by concrete crushing in compression and/or reinforcement yielding in tension. Both of these phenomena can be well modeled by plasticity. If a section is exposed to further deformation upon reaching the load capacity, the load drops, but usually not immediately to a zero value. The post-failure response is often dominated by highly ductile behavior of the reinforcing bars, which can typically sustain strain up to the order of $10^{-1}$. The corresponding residual load carried by a failed section or joint cannot be neglected when analyzing the disintegration and dynamic motion of a structure during demolition. In tension, the behavior of reinforcing bars can still be modeled by simple one-dimensional plasticity, but in compression, plastic buckling has to be taken into account. The present paper deals with modeling of the latter phenomena.

\section{Physical phenomena}

Let us consider an R/C member which is loaded by such a combination of axial force and bending moment, that at least on one side it is exposed to compression. Concrete crushing starts when the maximum compressive strain attains the level of roughly 0.002 . As the crushed concrete spalls, it exposes longitudinal reinforcing bars. Upon losing the support of concrete cover, the reinforcing bars buckle outward Fig. 1. The buckling length is limited either by transversal stirrups or by the length of the spalled concrete zone. Eventually the bars yield in plastic hinges, which form in the locations of extensive bending. Since the crushed and spalled concrete ceases to contribute to the load bearing ability of the section, its overall response is then dominated by the behavior of the buckling bars.

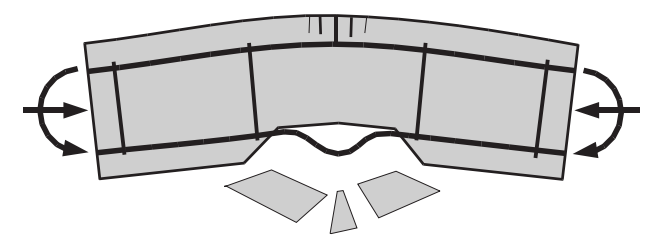

Fig. 1: Concrete cover spalling and reinforcement buckling in an $\mathrm{R} / \mathrm{C}$ member

\section{Analytical model}

\subsection{Loading and boundary conditions}

The behavior of a buckled bar is modeled by the relationship between applied axial force $P$ and the relative displacement of its ends $u$, as shown in Fig. 2. Force $P$ is positive when compressive and similarly positive $u$ means contraction. We 
a)

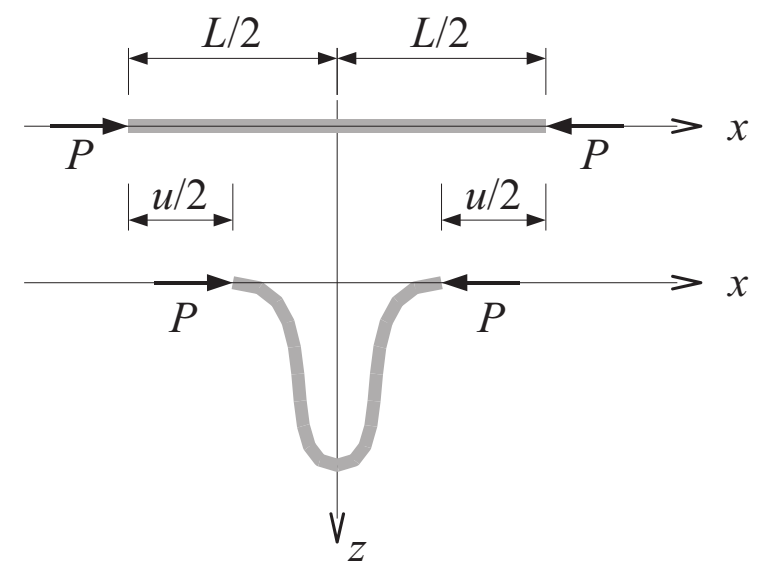

Fig. 2: Configuration and loading of a reinforcing bar: a) undeformed, b) deformed

consider that the bar is free to buckle in plane $x$ - $z$ within length $L$. As the bar is in reality continuous, the in-plane rotations and displacements in $z$-direction are fixed at the end points of length $L$. Since the problem is symmetric about axis $z$, we solve it only on one half of $L$, i.e. in the interval $\langle-L / 2,0\rangle$. Consequently, the displacement in $x$-direction and the rotation are fixed on the symmetry axis.

\subsection{Assumptions}

We accept the following general assumptions:

(1) The bar is modeled as a Bernoulli-Euler beam, i.e. the planar cross-sections perpendicular to the central line prior to deformation remain so also after deformation takes place. This assumption is acceptable, since the length of the bar, which is free to buckle, is usually much larger than the cross-sectional size.

(2) The bar undergoes finite displacements, therefore the equilibrium equations are formulated on the deformed configuration.

(3) In the post-buckling regime, the elastic deformations are negligibly small compared to the plastic deformations. Consequently material is modeled as rigid-plastic with linear hardening. Since we do not consider load reversals, we will use the deformation theory of plasticity.

(4) When the bar buckles, the contribution of central-line axial contraction to the overall contraction is negligible in comparison with the contribution due to bending (finite deflection).

\subsection{Moment-curvature relation}

In the view of Assumption (1), the normal strain distribution is linear on the bar cross-section. Since axial straining of the central line is neglected [Assumption (4)], the strain can be expressed as:

$$
\varepsilon=\zeta \cdot \kappa,
$$

where $\zeta$ denotes the local axis normal to and originating at the central line, and $\kappa$ is the curvature of the central line.

Assumption (3) implies that the material constitutive law can be written as:

$$
\begin{array}{lll}
\varepsilon=0 & \ldots \ldots & \text { for }|\sigma|<\sigma_{y} \\
\sigma=\operatorname{sgn}(\varepsilon) \sigma_{y}+E_{h} \varepsilon & \ldots . . & \text { for }|\sigma| \geq \sigma_{y}
\end{array}
$$

where $\sigma$ is the normal stress, $\sigma_{y}$ is the yield strength and $E_{h}$ is the linear-hardening modulus.

By combining Eqs. (1) and (2) and considering the equivalence of bending moment $M$ and the moment due to normal stress, the following relation is obtained:

$$
M=\operatorname{sgn}(\kappa) M_{0}+K_{p} \kappa
$$

where

$$
M_{0}=\int_{A} \zeta \sigma_{y} \mathrm{~d} A
$$

and

$$
K_{p}=\int_{A} \xi^{2} E_{h} \mathrm{~d} A
$$

and $A$ is the cross-sectional area of the bar. Note that Eq. (3) holds only for those parts of the bar which are fully plastic (plastic hinges), i.e. $|\sigma| \geq \sigma_{y}$ on the entire section, or, in terms of the bending moment, $|M| \geq M_{0}$. Outside the plastic hinges, the curvature is zero for any $M$.

\subsection{Equilibrium on a deformed bar}

Let us consider a symmetric half of a buckled bar, as shown in Fig. 3. The maximum deflection is denoted as $w$. To satisfy

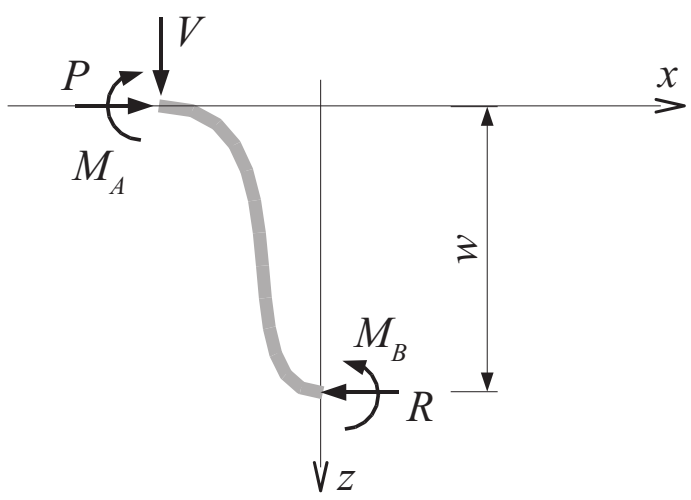

Fig. 3: Load and reactions on a deformed bar

the equilibrium of forces in $x$ and $z$ directions, reaction $R=P$ and $V=0$. The anti-symmetry of the deformed shape implies that moment reactions $-M_{A}=M_{B}$. To maintain the equilibrium of moments, we require

$$
2 M_{B}=P w .
$$

It is obvious from Fig. 3 and Fig. 4 that the bending moment induced by the load and the reactions is a linear function of coordinate $z$ :

$$
M=-M_{B}+P z
$$

which in combination with Eq. (6) gives:

$$
M=P\left(z-\frac{w}{2}\right) .
$$

\subsection{Geometry of a deformed bar}

As discussed in section 3.3, the curvature of the deformed bar outside the plastic hinges is zero, which means that the corresponding part of the central line remains straight.

The shape of the central line within the plastic hinges can in general be obtained by solving together Eqs. (3) and (8). 


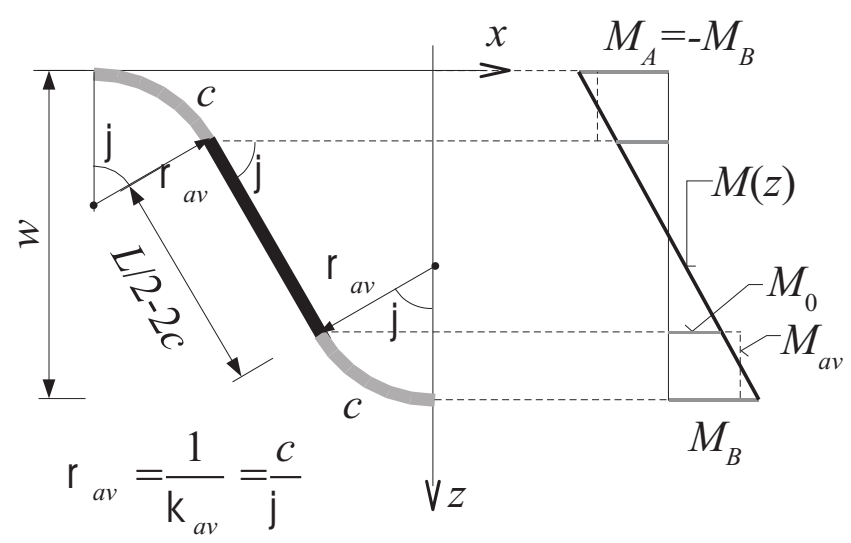

Fig. 4: Geometry and distribution of bending moment on a deformed bar

However, solution of the differential equation is rather complicated, since it involves elliptic integrals [3].

To simplify the problem, we approximate the shape of the central line in plastic hinges by circular arches with constant curvature $\kappa_{a v}$ (grey parts in Fig. 4). Due to the anti-symmetry, the plastic hinges on both ends of the analyzed half of the bar have the same length $c$. The arches are delimited by angle $\varphi$. Then

$$
\kappa_{a v}=\frac{\varphi}{c} .
$$

To ensure a smooth transition from the plastic hinges to the straight rigid portion of the bar (black part in Fig. 4), the latter has to be inclined at the same angle $\varphi$ from the horizontal direction. While taking into account Assumption (4) (the central line length remains constant and equal to $L / 2$ ), the two parameters $c$ and $\varphi$ completely describe the shape of the deformed central line. Then it is obvious from Fig. 4 that maximum deflection $w$ can be expressed as:

$$
w=2 \frac{c}{\varphi}(1-\cos \varphi)+\left(\frac{L}{2}-2 c\right) \sin \varphi
$$

Similarly, the relative displacement of the bar ends (Fig. 2) is:

$$
u=2\left\{\frac{L}{2}-\left[2 \frac{c}{\varphi} \sin \varphi+\left(\frac{L}{2}-2 c\right) \cos \varphi\right]\right\} .
$$

\subsection{Derivation of $P$ - $u$ relationship}

In the light of Eq. (3), the assumption of constant curvature implies that the bending moment within a plastic hinge is also constant. Thus, for the right-side hinge:

$$
M_{a v}=M_{0}+K_{p} \kappa_{a v} .
$$

We consider that this constant moment is also equal to the average moment in the plastic hinge (Fig. 4), then

$$
M_{a v}=\frac{1}{2}\left(M_{0}+M_{B}\right) \text {. }
$$

Now we substitute equations in the following order: $(9) \rightarrow(12) \rightarrow(13)$, from which we express $M_{B}$. This result is substituted together with Eq. (10) into Eq. (6), which allows us to express force $P$ in terms of $c$ and $\varphi$ :

$$
P=\frac{2 M_{0}+4 K_{p} \frac{\varphi}{c}}{2 \frac{c}{\varphi}(1-\cos \varphi)+\left(\frac{L}{2}-2 c\right) \sin \varphi} .
$$

In order to eliminate the length of the plastic hinge $c$, we consider that the bending moment from Eq. (8) must be equal to $-M_{0}$ at the right end of the left-side hinge, i.e. for

$$
z=\frac{c}{\varphi}(1-\cos \varphi)
$$

Eq. (8) is then rewritten in the form:

$$
-M_{0}=P\left[\frac{c}{\varphi}(1-\cos \varphi)-\frac{w}{2}\right] .
$$

After substituting Eqs. (10) and (14), the above equation is solved for $c$. Of two roots, the following one is relevant:

$$
\begin{aligned}
c & =\frac{\sqrt{2 K_{p} \varphi^{2} \sin \varphi\left(L M_{0}-L M_{0} \cos \varphi+2 K_{p} \varphi^{2} \sin \varphi\right)}}{2 M_{0}(1-\cos \varphi)} \\
& -\frac{2 K_{p} \varphi^{2} \sin \varphi}{2 M_{0}(1-\cos \varphi)} .
\end{aligned}
$$

If we now substitute Eq. (17) into Eqs. (11) and (14), both $P$ and $u$ depend only on parameter $\varphi$. The desired $P-u$ relationship is thus obtained in a parametric form. Note that parameter $\varphi$ has a clear physical meaning: it is the angle of inclination of the straight portion of the buckled reinforcing bar. Note also that the derived equations are valid only after the bar cross sections at $x=-L / 2,0, L / 2$ have completely yielded due to post-buckling bending.

\subsection{Extreme strain}

A complete rupture of the buckled bar is initiated when the extreme strain reaches material strain capacity $\varepsilon_{u}$. This first happens at the sections with extreme bending moments $M_{A}$ or $M_{B}$, that is, for $x=-L / 2,0, L / 2$. On these sections, the rupture criterion is first satisfied at the most distant points with $\zeta=\zeta_{\text {ext }}$. By using Eqs. (6), (14), (10), and (17) the extreme moment is expressed in terms of parameter $\varphi$. Then we obtain the extreme strain from Eqs. (3) and (1) as:

$$
\varepsilon_{\text {ext }}=\xi_{\text {ext }} \frac{M_{B}-M_{0}}{K_{p}} \text {. }
$$

\section{Validation}

In order to validate the proposed analytical model, a typical problem of a buckling bar is solved and the results are compared with those obtained with FEM.

The material and geometrical properties of the analyzed reinforcement bar are listed in Table 1 . They correspond to standard steel no. 10 216, which was in the past often used in precast R/C panels. For the sake of simplicity of the FE analysis, the cross section has the shape of a square with side $d$.

Table 1: Material and geometrical properties of the analyzed reinforcement bar

\begin{tabular}{|c|c|c|c|c|c|c|}
\hline$\left.E^{*}\right)$ & $\left.v^{*}\right)$ & $\sigma_{y}$ & $E_{h}$ & $\varepsilon_{u}$ & $L$ & $d$ \\
\hline $\mathrm{GPa}$ & - & $\mathrm{MPa}$ & $\mathrm{MPa}$ & - & $\mathrm{m}$ & $\mathrm{m}$ \\
\hline 210 & 0.3 & 206 & 1387.5 & 0.24 & 0.2 & 0.01 \\
\hline
\end{tabular}

$\left.{ }^{*}\right)$ Used only in FE analysis 
The FE analysis was preformed assuming plane stress, finite displacements and finite strains. The material was modeled as elastic-plastic with linear hardening. The model represented the entire length $L$. To facilitate buckling, the bar was given a piecewise linear lateral imperfection with the maximum value at the vertical symmetry line equal to $0.25 \%$ of $L$. The non-uniform FE mesh consisted of $100 \times 209$-noded elements, and it was refined in the locations of the plastic hinges.

According to Eq. (18), the bar rupture is initiated at $P=4.34 \mathrm{kN}$ and $u=0.0342 \mathrm{~m}$, which correspond to $\varphi=0.804962 \mathrm{rad}$. Fig. 5 compares the post-buckling load-displacement curves obtained with the proposed model and with FEM. A close agreement is evident for the entire relevant range, i.e. up to $u=0.0342 \mathrm{~m}$. Fig. 6 shows the values of extreme strain calculated according to Eq. (18) and the corresponding extreme values of logarithmic strains obtained with FEM. It is obvious that the proposed model captures very well the extreme compressive strain, while it overestimates the tensile strain. Note that the difference between the strains on the compressed and tensioned surfaces is a result of considering the finite displacements and strains in the FE analysis.

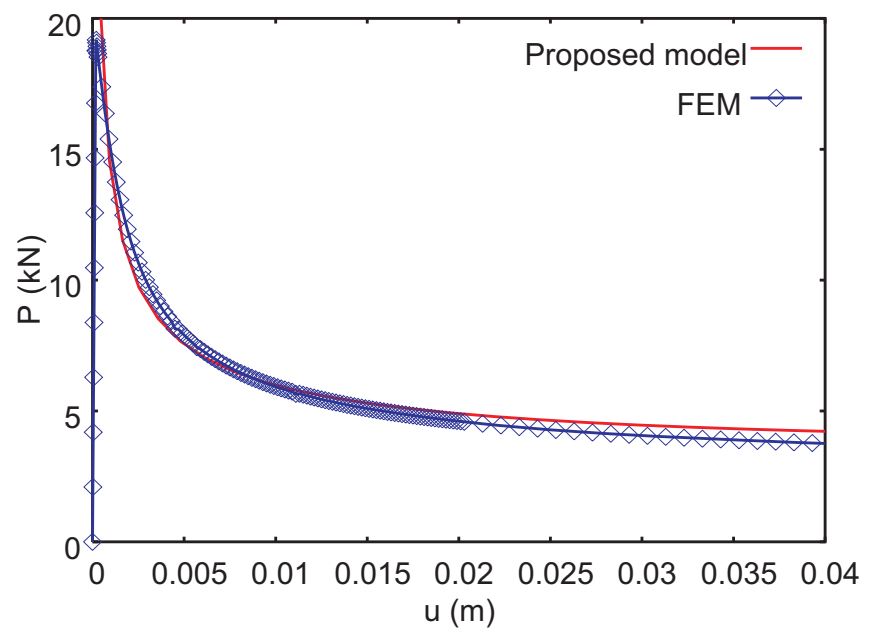

Fig. 5: Post-buckling load-displacement curves of a reinforcement bar

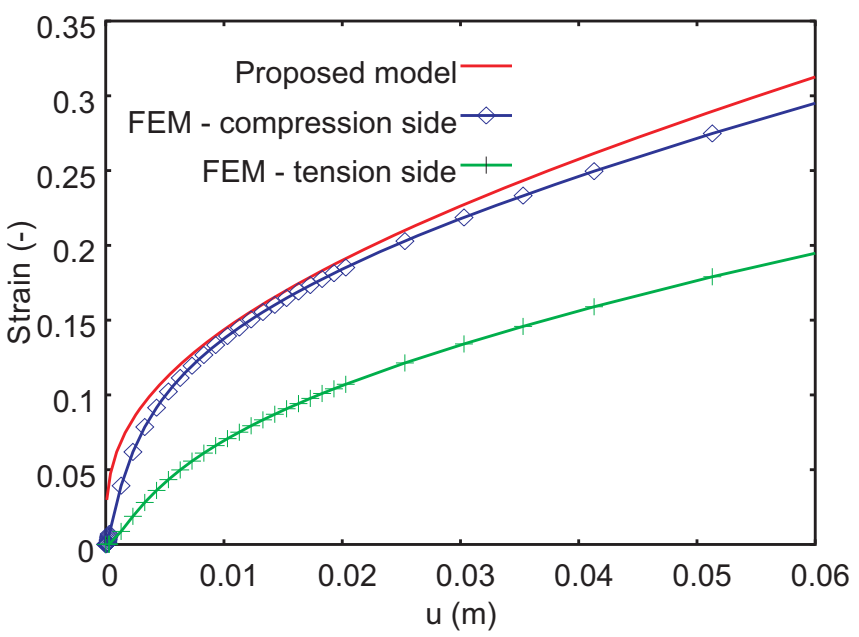

Fig: 6: Extreme strains vs. end displacement at $x=0$ and $\zeta= \pm d / 2$; absolute values of logarithmic strains are plotted as FEM results

\section{Concluding remarks}

A simplified approach to the analysis of the post-buckling behavior of a compressed bar has been presented. The simplification consists namely in considering rigid-plastic material behavior, neglecting axial contraction of the central line, and approximating the shape of the deformed central line in plastic hinges by a circular arch. Consequently, axial loading force $P$, end displacement $u$, and extreme strain $\varepsilon_{u}$ can be expressed in relatively simple closed forms. This feature is particularly desirable, since the formulation will be used in a multiscale context to model the behavior of an $\mathrm{R} / \mathrm{C}$ structure during demolition [2].

The results obtained with the proposed model show very close agreement with those obtained by a realistic detailed finite element analysis, which justifies the use of the simplifying assumptions.

\section{Acknowledgment}

The research presented in this paper was supported by grant no. 103/02/0658 provided by the Science Foundation of the Czech Republic and by Contract of the Ministry of Education of the Czech Republic No. J04/98: 210000003.

\section{References}

[1] Kabele P., Pokorný T.: "Computational Analysis of Precast Concrete Building Deconstruction." In "Proceedings of the Fifth World Congress on Computational Mechanics (WCCM V), July 7-12, 2002, Vienna, Austria" (Editors: H. A. Mang, F. G. Rammerstorfer, and J. Eberhardsteiner), Vienna University of Technology, Austria, http://wccm.tuwien.ac.at.

[2] Kabele P., Kalousková M.: "Multiscale Stochastic Simulation of Building Demolition." In Proceedings of the Sixth World Congress on Computational Mechanics (WCCM VI), to appear.

[3] Shames I. H., Dym C. L.: "Energy and Finite Element Methods in Structural Mechanics." Taylor \& Francis 1991.

Doc. Ing. Petr Kabele, Ph.D.

phone: 1420224354485

e-mail: petr.kabele@fsv.cvut.cz

Department of Structural Mechanics

Czech Technical University in Prague

Faculty of Civil Engineering

Thákurova 7

16629 Prague 6, Czech Republic 